\title{
The application of using TFEM to detect HC favorable area in buried-hills of Jizhong
}

\author{
YanliLi , QiangWei , YuqingWang, XuejunLiu, \\ ZhanxiangHe \\ BGP, CNPC \\ Zhuozhou, China
}

\author{
Fengming Jin \\ PetroChina Huabei Oilfield Company \\ Renqiu, China
}

\begin{abstract}
Because the buried hill is deeply buried and seismic data is difficult to identify, it is difficult to exploration the buriedhill in Jizhong Depression.Time-frequency electromagnetic method (TFEM) has the characteristics with directly detecting oil and gas reservoirs, and core drilling test and analysis results show that the buried hill in Jizhong depression has good physical condition. Through application of time-frequency electromagnetic exploration, the hydrocarbon anomaly information of buried hill is effectively extracted. Combined with the known wells and time-frequency electromagnetic anomalies of buried hill reservoir, we predicted hydrocarbon favorable area of buried hill in the study area to guide the oil and gas exploration. Good results have been achieved by this approach.
\end{abstract}

Keywords-time-frequencyelectromagnetic method; oiland gasprediction; buriedhill; JizhongDepression ;

\section{INTRODUCTION}

The oil and gas resources is rich in Jizhong depression, buried hills have a great potential for exploration. With the improvement of exploration degree, large scale and shallow buried hill that is easily identified by seismic data has been found and drilled. In recent years, a number of exploration wells, Wengu3, Chang3, Niudong 1 and so on, successfully drilled carbonate buried hill reservoir, which make the exploration area of buried hill expand continuously. These buried hill reservoirs that is deeply buried and difficult to identify attribute to subtle buried-hill reservoir ${ }^{[1]}$. The reflection information of this kind of buried hill is weak on seismic data ${ }^{[2]}$, the formation of petroleum accumulations is complex and diversity ${ }^{[3]}$, so it is difficult to explore the buried hill and drilling cost is high. The time-frequency electromagnetic method has the characteristics of low cost, directly detecting of oil and gas reservoirs, the technology has gradually mature after years of development, and a good exploration results have been achieved ${ }^{[4]}{ }^{[5]}$ in many oil fields at home and abroad. But time-frequency electromagnetic method is only be used to predict hydrocarbon potential of clastic rock in past. In this paper, time-frequency electromagnetic method is firstly applied to predict hydrocarbon potential of carbonate buried hill. In order to predict the hydrocarbon potential of deep-buried hills in Jizhong Depression, 30 lines, about $800 \mathrm{~km}$ of time-frequency electromagnetic exploration work have been carried out in in
Wen 'an slope and Dacheng uplift, and good results have been achieved.

\section{METHOD}

Through exciting by one excitation source, the time-frequency electromagnetic method can collect time-domain and frequency-domain signal at the same time. It changes the conventional electromagnetic methods which separately collect time-domain and frequency-domain signal, and combine time-domain with frequency-domain signal, so the efficiency of collection and detection effect is improved.

The construction method of TFEM in field was shown in figure 1.The transmitter that is a long wire electric dipole source (AB), is grounded by several parallel copper wires and a square-wave current is generated by a high-power generator with a preset frequency. The receiver is arranged at a certain distance from electric dipole source, offset distance is often several kilometers to more than 10 kilometers. The receivers receive the electric field signal paralleled $\mathrm{AB}$ by electrodes, magnetic field signal (Ex) by vertical bar magnet. The distance between station and station is $200 \mathrm{~m}$.

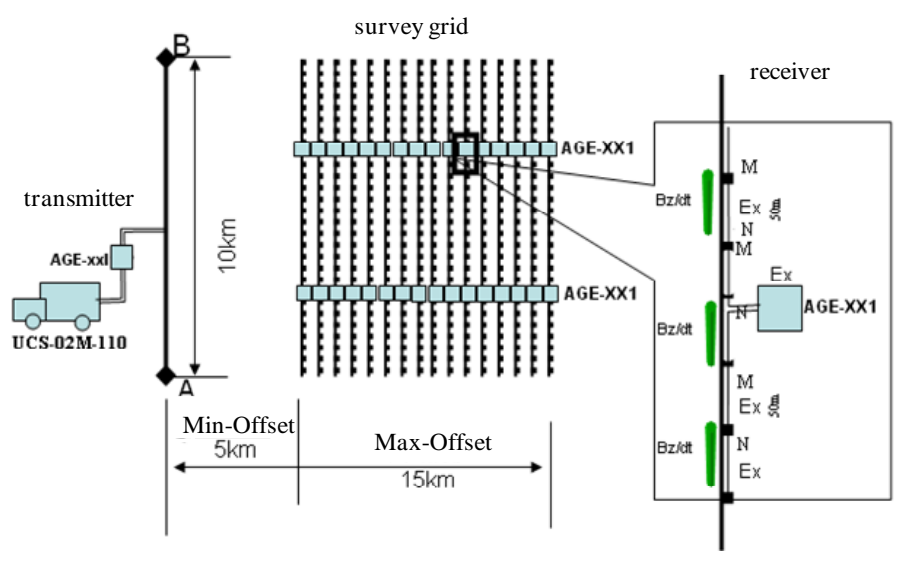

Fig. 1.Field layout of TFEM

Time and frequency electromagnetic method is a new type of electromagnetic prospecting technology, compared with the conventional method, it has four major advantages: (1) high power excitation, the power is up to $200 \mathrm{~kW}$ which is ten times higher than the power of conventional method; 
(2)detecting depth is deeper, and can effectively excite anomalies of sub crustal oil and gas;(3) time-domain and frequency-domain information is measured at the same time; (4)the polarizability and resistivity anomaly are both studied.

\section{DATA Collection, PRocessing AND}

\section{INTERPRETATION}

\section{A. Data Collection}

Because the buried hill is buried more than $4000 \mathrm{~m}$ in study area and shallow interference is strong, in order to obtain high precision data about the weak information of deep target, two data acquisition technology is took in field.

According to the characteristics with deeply buried hill, excitation cycle time is extended to 40s in field data collection in order to effectively obtain the information of buried hill. Because when the maximum excitation cycle is 32s, the target that buried $6000 \mathrm{~m}$ can be detected. In order to ensure that deep buried target in different electric conditions can be detected, excitation cycle time is extended to 40 s according to the simulation results.

According to the characteristics with strong shallow interference in the study area, a simple and effective way is applied to suppress random noise signals in the process of acquisition, by gathering more multicycle original signal, the amplitude of every frequency points is added in multicycle signal, then, the average value of the summation is made as the amplitude value of the signal. Because the background noise is the random noise signals, more multicycle signal can be collected to completely eliminate the influence of random noise signal, but if the acquisition cycle is too much, the construction efficiency will be seriously affected. According to the noise level in the study area, when the collection cycle number is 32, signal-to-noise has been increased from 2 times to 25 times, it is not only effectively suppress the influence of noise, but also approve the efficiency, high quality data is obtained.

\section{B. Data Processing}

In order to extract anomaly information of buried hill, constrained inversion method is adopted basing on the well (drilling, logging) and seismic model. The basic clue is setting a geological model (Fig.2.) and initial resistivity model (Fig.3.) according to the seismic and well date, then repeatedly finding geoelectric model that makes the forward curve and observation data curve fitting, when the fitting error is up to error standards, fitting calculation is stopped, the current geoelectric model is saved as the final inversion results (Fig.4.).By resistivity constrained inversion, resistivity background that reflects structure information of electromagnetic data can be obtained. The polarizability is obtained through constrained inversion of polarizability in every formation (Fig.5.). So that we can accurately separate resistivity information and polarization information (related to oil and gas, water) reflecting physical property of reservoir from the time-frequency data. By constraining inversion, electrical and polarization information can be correspond with formation, it is helpful to extract deep anomaly information, and physical property can be better reflected.

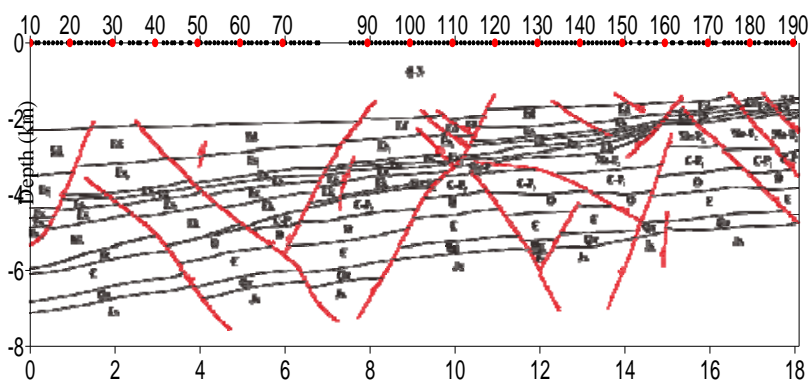

Fig. 2.Geological model of WD14E11 line

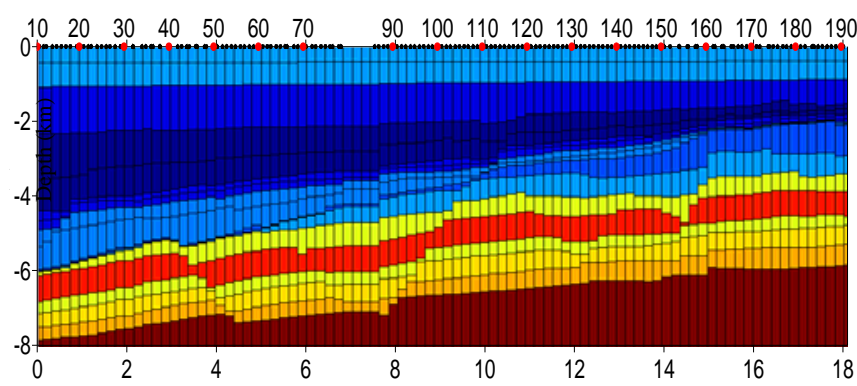

Fig. 3.Initial resistivity model of WD14E11 line

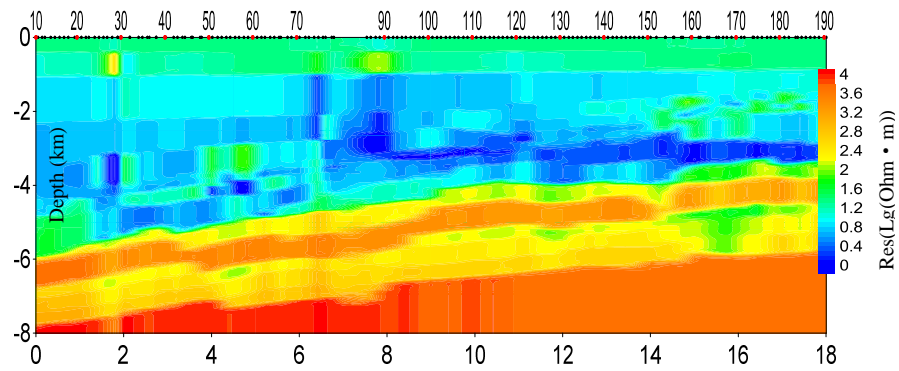

Fig. 4.Resistivity constrained inversion profile ofWD14E11 line

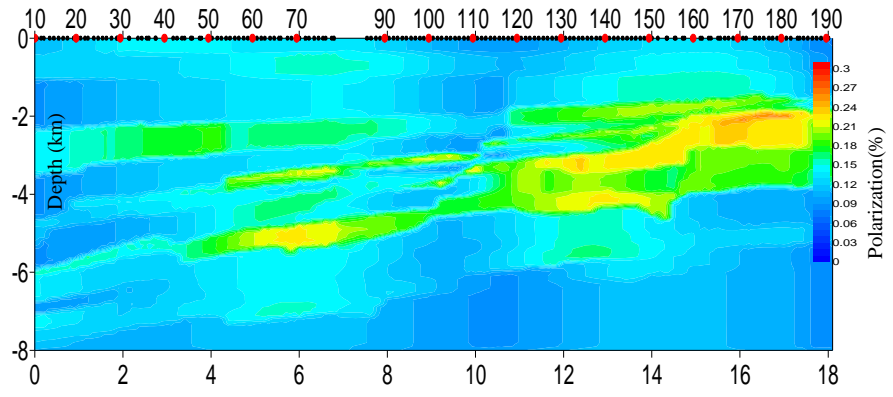

Fig. 5. Polarization anomaly profileofWD14E11 line

\section{Data Interpretation}

Induced polarization and electrical characteristics is the basis of data interpretation. Through core test and electric logging data of known buried hill reservoir, induced polarization and electrical characteristics of buried hill 
reservoir are studied, the results show that buried hill reservoir is characterized by high amplitude, high phase and medium - low resistivity anomaly, and with the higher oil saturation, the responding of the three parameters is stronger. There are good physical conditions to predict hydrocarbon potential of buried hill by using induced polarization and electromagnetic anomaly.

According to the physical properties of buried hill reservoir and combined with the result of known wells, the areas that are characterized by high polarization and low resistivity have been delineated as the favorable zones (Fig.6).

Two wells were deployed according to other data and because the time and frequency electromagnetic anomalies are weak, oil and gas have not been discovered, which confirmed that time and frequency electromagnetic method has negative effect, it has certain significance to reduce the risk of drilling.

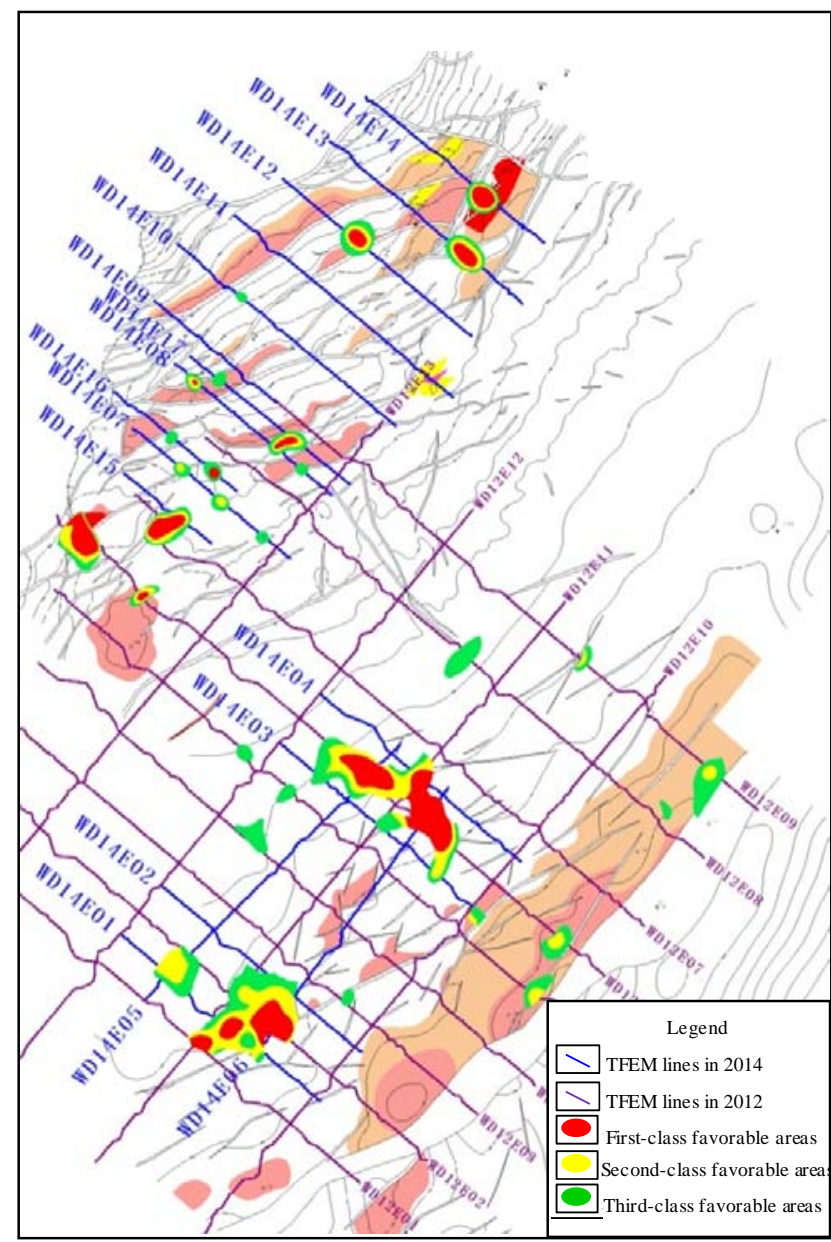

Fig. 6.Hydrocarbon favorable areas of buried hills in Wen 'an slope and Dacheng uplift (The base map is the structure map of Fujunshan Formation (Cambrian))

\section{CONCLUSIONS}

The application of time and frequency electromagnetic method in deep buried hill show that time and frequency electromagnetic anomaly information (resistivity and polarization) has high correlation with oil and gas, and can be considered as effective information to identify oil and gas. Combined with seismic, drilling, petroleum geology, it is a new ideas and method to predict favorable target, optimize and evaluate of buried hill, especially significant to subtle buried-hill reservoir.

\section{REFERENCES}

[1] Zhao Xianzheng, Wang Quan, Jin Fengming, et al. Main controlling factors and exploration practice of subtle buried-hill hydrocarbon reservoir in Jizhong Depression. Acta Petrolei Sinica, 2012, 33 (s1) :71-79.

[2] Chen Qinghua, Lao Haigang, Wu Kongyou, et al. Favorable hydro-cabon accumulation conditions for carbonate reservoirs in deep-buried hills in the Jizhong Deprssion, Bohai Bay Basin. NaturalGas Industry, 2013, 33 (10):32-39.

[3] Gao Xianzhi, Wu Weitao, Lu Xuejun,etal. Multiplicity of hy-drocarbon reservoir and accumulation controlling factors within buried hills in Jizhong Depression[J],Journal of China University of Petroleum: Edition of Natural Science,2011,35(3):31-35.

[4] TFEM applications in the Block A,Niger.Sun Zhihua,Fu Jilin,Yang Shujiang et al.OGP,2005,40(1):147-151.

[5] Luo Weifeng,He Zhanxiang,Wangcaifu et al.A test study of induced polarization effects of magnetotelluric in oil and gas detection. OGP, 2011,6 (46): :978-983. 